\title{
Role of metformin in lung cancer treatment and prevention: Are we any closer to the answer?
}

\author{
Samuel Kim, MD
}

From the Department of Surgery, University of Arizona, Tucson, Ariz.

Disclosures: Author has nothing to disclose with regard to commercial support.

Received for publication March 28, 2016; accepted for publication March 30, 2016

Address for reprints: Samuel Kim, MD, Department of Surgery, University of Arizona, 1501 N Campbell Ave,

Room 4302, Tucson, AZ 85724 (E-mail: eskim@surgery.arizona.edu).

J Thorac Cardiovasc Surg 2016;152:62-3

$0022-5223 / \$ 36.00$

Copyright (c) 2016 by The American Association for Thoracic Surgery

http://dx.doi.org/10.1016/j.jtcvs.2016.03.072

Since an observational study published in $2005^{1}$ suggesting an association between the use of metformin and decreased risk of any cancer, there has been an explosion of preclinical and clinical studies examining metformin's antineoplastic activity against various cancers and the potential mechanism for this activity. Inhibition of mammalian target of rapamycin complex 1 (mTORC1), which plays a pivotal role in the metabolism, growth, and proliferation of cancer cells, has been proposed as a potential anticancer mechanism of metformin. ${ }^{2,3}$ Numerous early-stage clinical trials are currently underway to investigate metformin's potential to prevent an array of cancers, including colorectal, prostate, endometrial, and breast cancers; however, recent observational studies examining the association between metformin use and survival in diabetic patients with advanced-stage non-small cell lung cancer (NSCLC) have demonstrated conflicting results, ${ }^{4,5}$ and there is a lack of clinical data assessing the antineoplastic effect of metformin in patients with NSCLC.

The article by Medairos and colleagues aims to take a step toward providing clinical data to address this question. ${ }^{6}$ In their retrospective analysis of 215 patients with stage I and II NSCLC who underwent pulmonary resection, progression-free survival (PFS) and overall survival were compared in patients with type II diabetes exposed to metformin (cohort $\mathrm{A}$ ), those with type II diabetes without metformin exposure (cohort B), and those without diabetes (cohort $\mathrm{C}$ ). The authors found a higher PFS in cohort A compared with cohort $\mathrm{B}$ and cohort $\mathrm{C}$ $(P=.02$ and .017 , respectively). Both multivariate analysis and inverse propensity-weighted analysis, after adjusting for age, sex, and $\mathrm{T}$ and $\mathrm{N}$ stage, demonstrated that metformin use in patients with type II diabetes was significantly associated with PFS. Overall survival did not differ among the 3 cohorts.

The study by Medairos and colleagues has several limitations that are worth highlighting. First, the study had a small number of cohorts, with a significant number of patients who were lost to follow up in both the diabetic and nondiabetic group (37 of 106 patients and 32 of 52 patients,

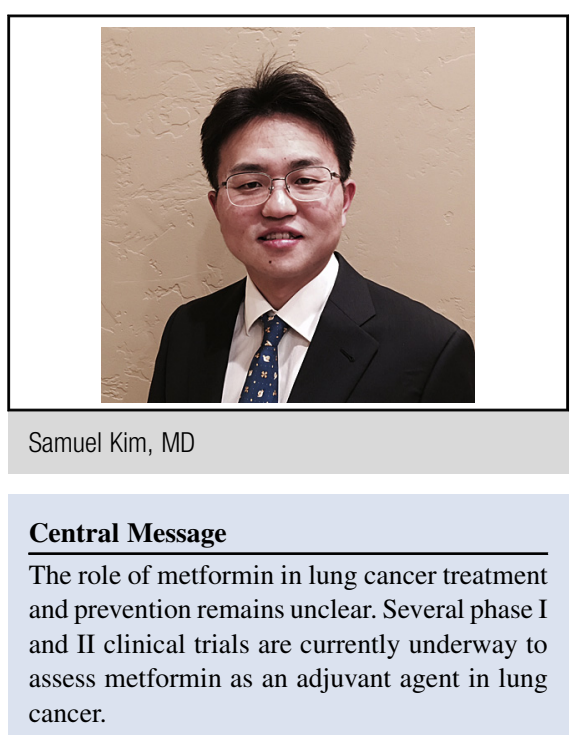

See Article page 55.

respectively), thus increasing the chance of introducing selection bias. Moreover, the duration of follow-up was relatively short (19 months). Second, the study is a retrospective review of cohorts. The use of metformin and other diabetes medications was determined solely from medical records, and no data on medication adherence were available. Finally, even though no statistical difference was achieved, the non-metformin group had a tendency toward higher-stage patients with more N1 nodes $(P=.09)$; thus, the disease-free survival could have been skewed.

Despite its limitations, the article by Medairos and colleagues provides yet more data connecting metformin exposure and improvement in survival for lung cancer in diabetic patients. Whether a clinical dose of metformin used to treat diabetes will have any impact on prevention and treatment of lung cancer remains far from clear, however. Virtually all of the laboratory studies reported to date used drug concentrations as much as 100-fold higher than those found in the serum of diabetic patients. Moreover, some have argued that a careful assessment of the observational studies conducted to date point to some important time-related biases that systematically exaggerated the reported antitumor effects of metformin. ${ }^{7}$ Several phase I and II clinical trials, including NRG-LU001, are currently underway to assess metformin as an adjuvant agent in lung cancer. The results hopefully will shed light on this controversy in the near future. 


\section{References}

1. Evans JM, Donnelly LA, Emslie-Smith AM, Alessi DR, Morris AD. Metformin and reduced risk of cancer in diabetic patients. BMJ. 2005;330:1304-5.

2. Chiang GG, Abraham RT. Targeting the mTOR signaling network in cancer. Trends Mol Med. 2007;13:433-42

3. Kasznicki J, Sliwinska A, Drzewoski J. Metformin in cancer prevention and therapy. Ann Transl Med. 2014;2:57.

4. Tan BX, Yao WX, Ge J, Peng XC, Du XB, Zhang R, et al. Prognostic influence of metformin as first-line chemotherapy for advanced non-small cell lung cancer in patients with type 2 diabetes. Cancer. 2011;117:5103-11.
5. Lin JJ, Gallagher EJ, Sigel K, Mhango G, Galsky MD, Smith CB, et al. Survival of stage IV lung cancer patients with diabetes treated with metformin. Am J Respir Crit Care Med. 2015;191:448-54.

6. Medairos RA, Clark J, Holoubek S, Kubasiak JC, Pithadia R, Hamid F, et al Metformin exposure is associated with improved progression-free survival in diabetic patients following resection for early-stage non-small cell lung cancer. J Thorac Cardiovasc Surg. 2016;152:55-61.e1.

7. Suissa S, Azoulay L. Metformin and the risk of cancer: time-related biases in observational studies. Diabetes Care. 2012;35:2665-73. 